Research Paper

\title{
A Network Meta-analysis Comparing the Efficacy and Safety of Anti-PD-1 with Anti-PD-L1 in Non-small Cell Lung Cancer
}

\author{
Wei You ${ }^{\bowtie}$, Mei Liu1 ${ }^{*}$, Ji-Dong Miao1, Yu-Qian Liao², Yi-Bing Song1, Dian-Kun Cai1 ${ }^{1}$, Yang Gao1, Hao Peng ${ }^{3}$ \\ 1. Department of Oncology, Zigong NO.4 People's Hospital, Zigong, Sichuan Province, 643000, People's Republic of China. \\ 2. Department of Medical Oncology, Jiangxi Cancer Hospital, Nanchang, Jiangxi Province, 330029, People's Republic of China. \\ 3. Department of Radiation Oncology, Sun Yat-sen University Cancer Center, People's Republic of China. \\ *Wei You and Mei Liu contributed equally to this work. \\ $\square$ Corresponding author: Professor Wei You, Department of Oncology, Zigong NO.4 People's Hospital, Zigong, Sichuan Province, 643000, People's Republic of \\ China. Telephone: +86-0813-8125329; Fax: +86-0813-2202665; E-mail: youweizg@126.com \\ (C) Ivyspring International Publisher. This is an open access article distributed under the terms of the Creative Commons Attribution (CC BY-NC) license \\ (https://creativecommons.org/licenses/by-nc/4.0/). See http://ivyspring.com/terms for full terms and conditions.
}

Received: 2017.08.14; Accepted: 2017.11.29; Published: 2018.03.10

\begin{abstract}
Background: This network meta-analysis aimed at comparing anti-programmed death 1 (anti-PD-1) with anti-programmed death ligand 1 (anti-PD-LI) immunotherapy in patients with metastatic, previously treated non-small cell lung cancer (NSCLC) who failed first-line treatment.

Methods: We searched electronic databases to identify all eligible clinical trials. End-points included overall survival (OS), progression-free survival (PFS) and objective response. Hazard ratios (HRs) or odds ratios (ORs) and corresponding $95 \%$ confidence intervals $(\mathrm{Cls})$ were extracted. Network meta-analysis was performed using the frequentist approach for multiple treatment comparisons.

Results: In total, 3024 patients were randomly assigned: 1117 received anti-PD-1 therapy (nivolumab + pembrolizumab), 569 received anti-PD-L1 (atezolizumab) and 1338 received docetaxel. Anti-PD-1 (HR, $0.56 ; 95 \% \mathrm{Cl}, 0.48-0.66)$ and anti-PD-LI (HR, 0.64; 95\% Cl, 0.51-0.79) achieved better OS than docetaxel, and anti-PD-1 was superior to docetaxel in terms of PFS (HR, 0.75; 95\% Cl, 0.62-0.89). Moreover, anti-PD-1 achieved the highest effect on OS and PFS, with a P-score of $91.2 \%$ and $95.5 \%$, respectively. With regard to tumor response, anti-PD-1 group had a higher rate of responders than that in anti-PD-L1 $(\mathrm{HR}, 0.35 ; 95 \% \mathrm{Cl}, 0.19-0.65)$ and docetaxel $(\mathrm{HR}, 0.36 ; 95 \% \mathrm{Cl}, 0.25-0.52)$ groups. Undoubtedly, anti-PD-1 and anti-PD-L1 obtained less toxicity profile than docetaxel, and no significant difference was observed between anti-PD-1 and anti-PD-L1 groups.

Conclusions: Anti-PD-1 may be a better choice for patients with metastatic and previously treated NSCLC who failed first-line treatment in terms of the treatment ranking.
\end{abstract}

Key words: Non-small cell lung cancer; PD-1; PD-L1; immunotherapy, Nivolumab.

\section{Introduction}

Lung cancer is the leading cause of cancer death worldwide, among which the subtype of non-small cell lung cancer (NSCLC) accounts for almost $85 \%$ of all cases [1]. Outcomes for NSCLC are still unsatisfactory although many improvements have been achieved with the development and usage of targeted therapy for patients with amenable mutations [2-8]. For patients failing previously first-line treatment, systemic docetaxel has been recommended as the second line for recurrent or metastatic disease since $1999[9,10]$. Later on, many new medications including pemetrexed and erlotinib have been developed to compare with docetaxel [11, 12]; however, both failed to achieve better survival outcomes but better side-effect profile. Therefore, developing new agents with better efficacy and less toxicities is crucial for clinicians and patients.

Immunotherapy is a new treatment in cancer 
care, and targeting the programmed death 1 (PD-1) and programmed death-ligand 1 (PD-L1) pathway has been proven to be a promising therapeutic option for patients with recurrent or metastatic NSCLC [13-20]. Due to the superior overall survival and less toxicities achieved by nivolumab, pembrolizumab and atezolizumab when comparing with docetaxel, these drugs were approved by the Unite States Food and Drug Administration (FDA) as the standard care for patients with NSCLC who failed platinumcontaining chemotherapy. Despite the similar efficacy, they have different antitumor mechanisms; nivolumab and pembrolizumab target PD-1 while atezolizumab targets PD-L1. Besides the blocking effect on PD-1 and PD-L1 interaction which could reactive suppressed immune cells to eliminate tumor cells [18, 21, 22], atezolizumab also blocks PD-L1 and B7-1 binding which results in stronger immune response [23-26]. Therefore, there comes a concern for clinicians that which kind of immunotherapy is better? However, no study to date has been carried out to characterize this issue. Therefore, we conducted this network meta-analysis to compare the therapeutic gain achieved by anti-PD-1 (nivolumab + pembrolizumab) and anti-PD-L1 (atezolizumab) immunotherapy in patients with locally advanced or metastatic, previously treated NSCLC who failed first-line therapy.

\section{Materials and Methods}

\section{Literature Search Strategy and Study Inclusion}

We searched the electronic databases of PubMed, Web of Science, Cochrane Library as well as WangFang database and National Knowledge Infrastructure to identify all eligible clinical trials regarding NSCLC. Supplementary Methods presented the detailed information on literature search strategy and study inclusion criteria for this network metaanalysis. Notably, there are main three treatment arms: anti-PD-1, anti-PD-L1 and docetaxel; each arm should only contain one medication.

\section{Data extraction}

Three investigators (W.Y, M.L and J.D.M) assessed the quality of included trials independently by examining the randomization, procedure, sample size estimation, adoption of blinding in study design, allocation concealment, whether intention-to-treat analysis was followed, loss to follow-up and dropout according to the Jadad/Oxford quality scoring system [27]. Another three investigators (Y.B.S, D.K.C and Y.Q.L) reviewed the included studies and extracted the data independently. Data on study design, study time, number of patients, randomization scheme, follow-up duration, treatment protocol, end-points and failure patterns were abstracted. Any discrepancies in quality assessment and data extraction were resolved by consensus.

\section{Statistical analysis}

The primary end-point was overall survival (OS), defined as the duration from randomization to death from any cause. Second endpoints included progression-free survival (PFS, defined as time from randomization to the first occurrence of disease progression) and objective response (complete response, partial response, stable disease, progressive disease). Patients with complete or partial response would be grouped as responders and those with stable or progressive disease as non-responders. Survival data were expressed as hazard ratio (HR) and objective response as odds ratio (OR). Traditional direct meta-analysis was conducted first using Stata 13.0 (StataCorp LP, College Station, TX, USA). We calculated the pooled estimates of HRs or ORs and corresponding 95\% confidence intervals (CIs) of direct comparisons between two therapeutic regimens. A two-sided $P$-value of $<0.05$ was considered significant. Heterogeneity across studies was tested by $X^{2}$ test and $I^{2}$ statistic along with a forest plot. Statistically significant heterogeneity was defined as a $X^{2} P$-value $<0.1$ or an $I^{2}$ statistic $>50 \%$.

Network meta-analysis was performed with $\mathrm{R}$ software (version 3.3.3; R Foundation, Vienna, Austria) using the netmeta package [28, 29] and a frequentist approach [28]. Treatment effects were estimated by calculating HRs or ORs with corresponding 95\% CIs. Heterogeneity or inconsistency between and within designs was assessed using the $\mathrm{Q}$ test, which was proposed to be a generalization of Cochran's test by Rücker et al. [28]. If no heterogeneity existed $(P>0.1)$, a fixed-effects model was used. In case of significant heterogeneity, use of a random-effects model and sensitivity analysis were considered. The P-score, proposed by Rücker and Schwarzer [30] as a frequentist similar to surface under the cumulative ranking curve [31, 32], was adopted to rank treatment arms. The P-score of the best treatment is $100 \%$, and the worst, $0 \%$. Overall grade 3-5 toxicities were compared using the $x^{2}$ test and a two-sided $P$-value of $<0.05$ was considered significant. Survival and objective response analysis were conducted in intention-to-treat population and toxicity comparison in patients receiving at least one dose of treatment. More details of the network meta-analysis are presented in the Supplementary Methods. 


\section{Results}

\section{Eligible studies}

Up to the June 30, 2017, we identified 6 potentially eligible studies $[13-15,17,19,20]$ in total (Figure S1). The study by Reck et al. [20] involving patients having previously untreated NSCLC with PD-L1 expression on at least $50 \%$ of tumor cells was excluded. Therefore, 5 studies [13-15, 17, 19] were included in this network meta-analysis, among which $2[13,14], 1[17]$ and $2[15,19]$ trials compared nivolumab, pembrolizumab and atezolizumab with docetaxel, respectively. Notably, the study by Herbst et al. [17] consisted of three treatment arms (i.e., pembrolizumab $2 \mathrm{mg} / \mathrm{kg}$, pembrolizumab $10 \mathrm{mg} / \mathrm{kg}$, docetaxel $75 \mathrm{mg} / \mathrm{m}^{2}$ ). The basic information of the 5 studies were presented in Table 1. Overall, 3024 patients were randomly assigned: 1117 received anti-PD-1 therapy, 569 received anti-PD-L1 therapy and 1338 received docetaxel. HRs and corresponding 95\% CIs of OS and PFS were obtained directly from the original text. Quality assessment of included trials is summarized in Table S1.

\section{Direct meta-analysis of OS and PFS}

Results of direct meta-analysis were presented in Figure 1. Heterogeneity was found in comparing anti-PD-1 with docetaxel for PFS, and random-effect model was therefore applied. Undoubtedly, anti-PD-1 (HR, 0.66; 95\% CI, 0.59-0.73) and anti-PD-L1 (HR, 0.73; 95\% CI, 0.62-0.84) immunotherapy were significantly better than docetaxel in improving OS. Moreover, anti-PD-1 was found to achieve better PFS (HR, 0.80; 95\% CI, 0.68-0.93); however, PFS was comparable between anti-PD-L1 and docetaxel (HR, 0.95; 95\% CI, 0.82-1.07).

\section{Network meta-analysis of OS and PFS}

The network plot of the three treatment arms (anti-PD-1, anti-PD-L1 and docetaxel) was shown in Figure S2. Docetaxel was established as the reference group in this network meta-analysis since it's the controlled arm in these five trials. No heterogeneity was detected within studies and fixed-effect model was employed. Results of multiple treatment comparisons were summarized in Table 2. Consistent with the results of direct meta-analysis, anti-PD-1 (HR, 0.56; 95\% CI, 0.48-0.66) and anti-PD-L1 (HR, 0.64; 95\% CI, 0.51-0.79) immunotherapy could significantly decrease overall death, and anti-PD-1 (HR, 0.75; 95\% CI, 0.62-0.89) even achieved better PFS compared with docetaxel. Intriguingly, no significant difference was found between anti-PD-1 and anti-PD-L1 with regard to OS (HR, 1.14; 95\% CI, 0.87-1.49) and PFS (HR, 1.24; 95\% CI, 0.91-1.69) (Figure 2). The corresponding P-scores for docetaxel, anti-PD-1 and anti-PD-L1 were $0,91.2 \%$ and $58.5 \%$ for OS, and $13.5 \%, 95.5 \%$ and $41.0 \%$ in terms of PFS, respectively. This indicated that anti-PD-1 achieved the best effect on both OS and PFS.

\section{Network meta-analysis of objective response}

In total, the responders and non-responders in anti-PD-1, anti-PD-L1 and docetaxel groups were 209 and 908, 79 and 490, 190 and 1148, respectively. Results of network meta-analysis were presented in Table 3 and Figure S3. Generally, the anti-PD-1 group obtained a higher rate of responders compared with docetaxel (OR, 0.36; 95\% CI, 0.25-0.52) and anti-PD-L1 (OR, 0.35; 95\% CI, 0.19-0.65), and comparable results were observed between docetaxel and anti-PD-L1 groups (OR, 1.02; 95\% CI, 0.62-1.66). Also, anti-PD-1 achieved the highest effect on tumor response with a P-score of $99.9 \%$ (Table 3).

Table 1. Summary of the 5 studies comparing immunotherapy with docetaxel in patients with locally advanced or metastatic, previously treated non-small cell lung cancer.

\begin{tabular}{|c|c|c|c|c|c|c|c|c|}
\hline \multirow[t]{2}{*}{ Study } & \multirow[t]{2}{*}{$\begin{array}{l}\text { No. of } \\
\text { patients }\end{array}$} & \multirow[t]{2}{*}{ Study time } & \multirow[t]{2}{*}{ Immunotherapy } & \multirow[t]{2}{*}{ Chemotherapy } & \multicolumn{2}{|c|}{ Median overall survival (months) } & \multicolumn{2}{|c|}{$\begin{array}{l}\text { Median progression-free survival } \\
\text { (months) }\end{array}$} \\
\hline & & & & & Immunotherapy & Chemotherapy & Immunotherapy & Chemotherapy \\
\hline \multicolumn{9}{|l|}{ Nivolumab vs. Docetaxel } \\
\hline Borghaei et al. 2015 & 582 & 2012.11-2013.12 & $3 \mathrm{mg} / \mathrm{kg}$ every 2 weeks & $\begin{array}{l}75 \mathrm{mg} / \mathrm{m}^{2} \text { every } 3 \\
\text { weeks }\end{array}$ & 12.2 & 9.4 & 2.3 & 4.2 \\
\hline Brahmer et al. 2015 & 272 & 2012.10-2013.12 & $3 \mathrm{mg} / \mathrm{kg}$ every 2 weeks & $\begin{array}{l}75 \mathrm{mg} / \mathrm{m}^{2} \text { every } 3 \\
\text { weeks }\end{array}$ & 9.2 & 6.0 & 3.5 & 2.8 \\
\hline \multicolumn{9}{|l|}{$\begin{array}{l}\text { Pembrolizumab vs. } \\
\text { Docetaxel }\end{array}$} \\
\hline Herbst et al. 2016 & 1034 & 2013.08-2015.02 & $\begin{array}{l}\text { Arm 1: } 2 \mathrm{mg} / \mathrm{kg} \text { every } 3 \\
\text { weeks } \\
\text { Arm 2: } 10 \mathrm{mg} / \mathrm{kg} \text { every } 3 \\
\text { weeks }\end{array}$ & $\begin{array}{l}75 \mathrm{mg} / \mathrm{m}^{2} \text { every } 3 \\
\text { weeks }\end{array}$ & $\begin{array}{l}14.9 \text { for } \operatorname{arm} 1 \\
17.3 \text { for } \operatorname{arm} 2\end{array}$ & 8.2 & $\begin{array}{l}3.9 \text { for } \operatorname{arm} 1 \\
4.0 \text { for } \operatorname{arm} 2\end{array}$ & 4.0 \\
\hline \multicolumn{9}{|l|}{ Atezolizumab vs. Docetaxel } \\
\hline Fehrenbacher et al. 2016 & 287 & 2013.08-2014.03 & 1200 mg ever 3 weeks & $\begin{array}{l}75 \mathrm{mg} / \mathrm{m}^{2} \text { every } 3 \\
\text { weeks }\end{array}$ & 12.6 & 9.7 & 2.7 & 3.0 \\
\hline Rittmeyer et al. 2017 & 850 & 2014.03-2015.04 & 1200 mg ever 3 weeks & $\begin{array}{l}75 \mathrm{mg} / \mathrm{m}^{2} \text { every } 3 \\
\text { weeks }\end{array}$ & 12.6 & 8.9 & 2.8 & 4.0 \\
\hline
\end{tabular}


Overall survival

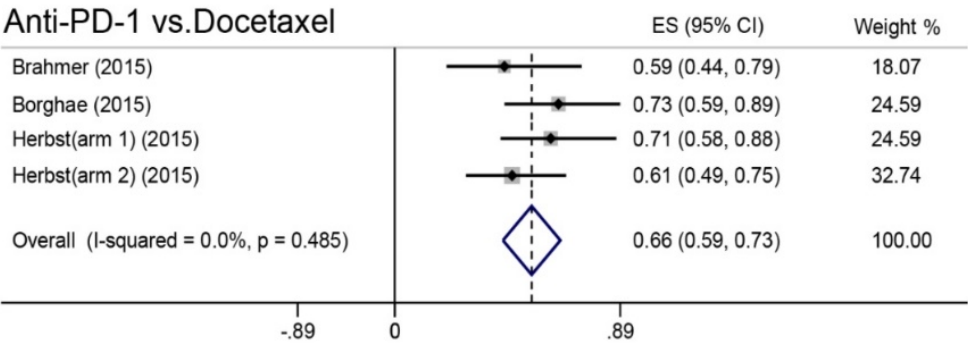

Anti-PD-L1 vs.Docetaxel

\begin{tabular}{|c|c|c|}
\hline Fehrenbacher (2016) & $0.73(0.53,0.99)$ & 22.80 \\
\hline Rittmeyer (2017) & $0.73(0.62,0.87)$ & 77.20 \\
\hline Overall $(I-$ squared $=0.0 \%, p=1.000)$ & $0.73(0.62,0.84)$ & 100.00 \\
\hline
\end{tabular}

Progression-free survival

Anti-PD-1 vs.Docetaxel

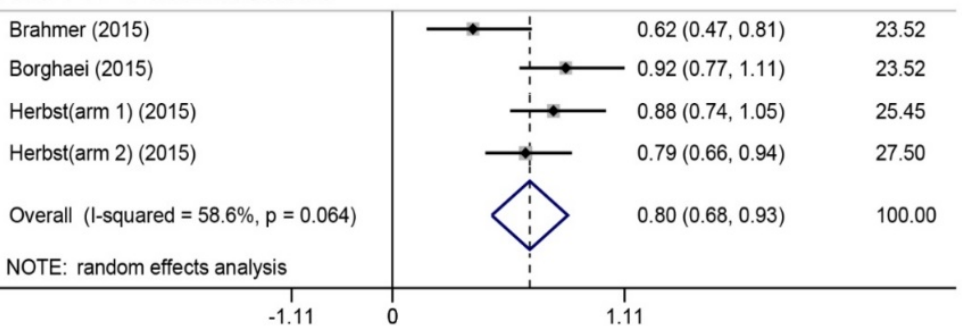

Anti-PD-L1 vs.Docetaxel

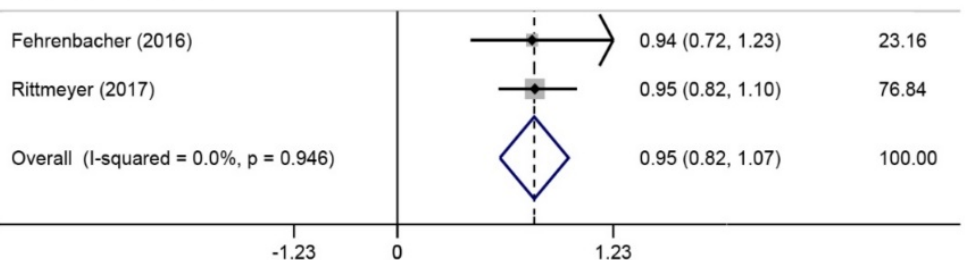

Figure 1. Forest plot of direct meta-analysis for overall survival and progression-free survival. PD-1 = programmed death 1; PD-LI = programmed death ligand 1; ES $=$ effect size; $\mathrm{Cl}=$ confidence interval.

Table 2. Summary of network meta-analysis results for overall survival and progression-free survival.

\begin{tabular}{|c|c|c|}
\hline Treatment arm & OS & PFS \\
\hline $\begin{array}{l}P \text { value of Overall } \\
\text { heterogeneity/inconsistency }\end{array}$ & 0.66 & 0.17 \\
\hline$P$ value of heterogeneity (within designs) & 0.66 & 0.17 \\
\hline$P$ value of heterogeneity (between designs) & / & / \\
\hline \multicolumn{3}{|l|}{ Docetaxel } \\
\hline $\mathrm{HR}$ & 1.00 & 1.00 \\
\hline P-score $(\%)$ & 0 & 13.5 \\
\hline \multicolumn{3}{|l|}{ Anti-PD-1 } \\
\hline HR $(95 \%$ CI $)$ & $0.56(0.48-0.66)$ & $0.75(0.62-0.89)$ \\
\hline P-score $(\%)$ & 91.2 & 95.5 \\
\hline \multicolumn{3}{|l|}{ Anti-PD-L1 } \\
\hline HR $(95 \%$ CI $)$ & $0.64(0.51-0.79)$ & $0.92(0.72-1.19)$ \\
\hline P-score (\%) & 58.8 & 41.0 \\
\hline
\end{tabular}

\section{Grade 3-5 toxicities}

The detailed number of patients experiencing grade 3-5 toxicity profiles in the study by Fehrenbacher et al. [15] and Rittmeyer et al. [19] were not available, and we therefore only compared the overall grade 3-5 toxicities between the three groups (Table 4). In total, 12.5\% (137/1100), 14.1\% (106/751) and $44.0 \%(624 / 1419)$ of patients in the anti-PD-1, anti-PD-L1 and docetaxel groups experienced grade 3-5 toxicities. The docetaxel group suffered significantly higher rate of grade 3-5 toxicities than anti-PD-1 $(P<0.001)$ and anti-PD-L1 $(P<0.001)$ groups, while it did not significantly differ between anti-PD-1 and anti-PD-L1 groups $(P=0.299)$. 


\section{Discussion}

Multiple treatment comparisons using network meta-analysis is a powerful method for comparing direct and indirect treatments in randomized clinical trials, and has been applied in head and neck cancers [33-35]. As far as we know, our network meta-analysis is the first study to compare the therapeutic gain achieved by docetaxel, anti-PD-1 and anti-PD-L1 immunotherapy in patients with locally advanced or metastatic, previously treated NSCLC who failed first-line treatment. Based on all available information extracted from included trials, we found that anti-PD-1 and anti-PD-L1 were much better than docetaxel in terms of OS, and anti-PD-1 achieved better PFS than docetaxel while no significant difference was detected between anti-PD-1 and anti-PD-L1 in terms of OS and PFS.

Due to the extremely poor survival of locoregionally advanced or metastatic, previously treated NSCLC who failed first-line therapy, purpose of treatment for this population was usually palliative. Therefore, efficacy as well as safety should be both considered when selecting therapy regimens. Although docetaxel has been established as the standard second-line care [9, 10], its efficacy is still unsatisfactory, with an median OS ranging from 6.0 to 9.7 months [13-15, 17, 19]. Moreover, the toxicity profile produced by docetaxel has also remained a crucial issue for both clinicians and patients. Therefore, it's urgently to develop new agents. Immunotherapy, main including blocking on PD-L1-PD-1 and PD-L1-B7-1 pathway [13, 14, 16, 17, 23, 26], has shown promising results for both better survival outcomes and less toxicities. In our current study, both direct and network meta-analysis consistently revealed anti-PD-1 and anti-PD-L1 significantly improved overall survival compared with docetaxel and these were also consistent with the results of primary studies [13-15, 17, 19]. The

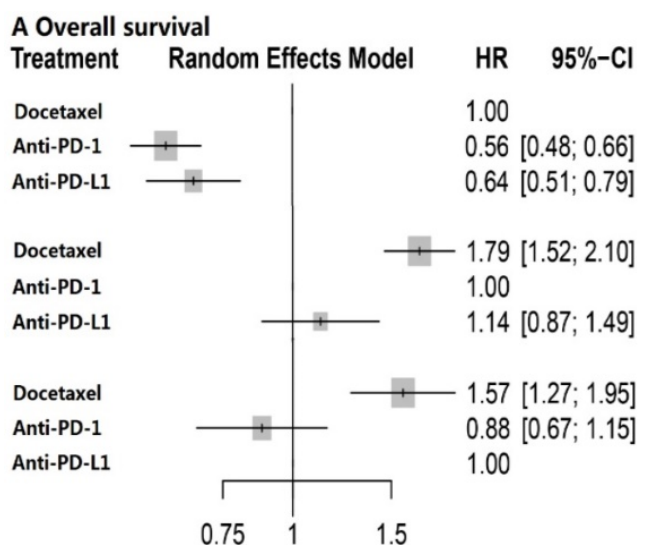

underlying reason for the OS benefit may be that response to treatment was more durable in the immunotherapy group than that in the docetaxel group.

PD-L1, an immune checkpoint protein expressed on both tumor cells and tumor-infiltrating immune cells, could mediate suppression of antitumor immunity by binding to its receptors PD-1 and B7-1 [36-38]. Therefore, anti-PD-L1 antibody like atezolizumab could block both PD-L1-PD-1 and PD-L1-B7-1 pathway which may produce stronger immune response than anti-PD-1 antibody which only blocks PD-L1-PD-1 binding [23-26]. Given this theoretical evidence, it may be reasonable to speculate that anti-PD-L1 antibodies should achieved better outcomes. However, clinical data obtained contradictory conclusion that anti-PD-1 achieved similar OS but better PFS than anti-PD-L1, and achieved the highest effect on OS and PFS with regard to P-score. These results indicated that the value of blocking PD-L1-B7-1 pathway may be further investigated in NSCLC. In the study by Brahmer et al. [14], PD-L1 expression was found to be neither prognostic nor predictive of efficacy in patients with squamous-cell NSCLC; however, it was associated with the benefit from anti-PD-1 treatment in non-squamous-cell NSCLC [13]. Therefore, it may be more appropriate to compare anti-PD-1 with anti-PD-L1 in highly selected patients based on the expression level of PD-L1 on tumor cells. Notably, in network meta-analysis, a probability of treatment ranking (P-score) would be produced even if the effect size among treatment arms was small and nonsignificant. It would be misleading if treatment decisions mainly depend on it. Therefore, in our study, both anti-PD-1 and anti-PD-L1 therapy were equally effective although anti-PD-1 achieved the highest P-score.

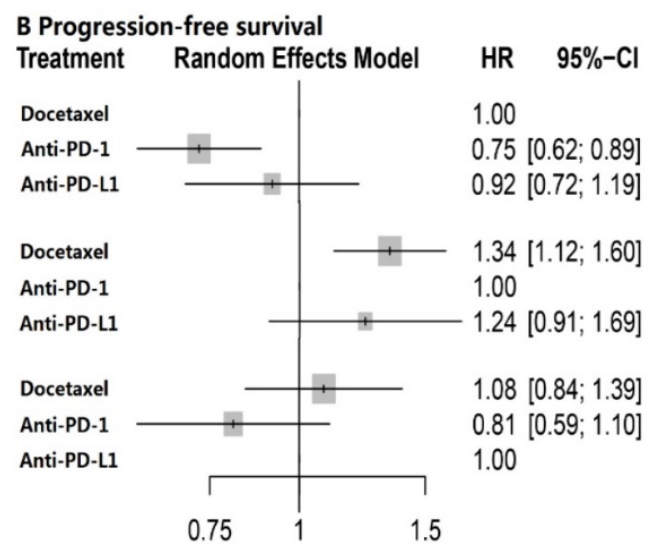

Figure 2. Forest plot of network meta-analysis for overall survival and progression-free survival with different reference groups. PD-1 = programmed death 1 ; $\mathrm{PD}-\mathrm{LI}=$ programmed death ligand $1 ; \mathrm{HR}=$ hazard ratio; $\mathrm{Cl}=$ confidence interval. 
Table 3. Summary of network meta-analysis results for tumor response.

\begin{tabular}{ll}
\hline Treatment arm & Response a \\
\hline$P$ value of Overall heterogeneity/inconsistency & 0.86 \\
$P$ value of heterogeneity (within designs) & 0.86 \\
$P$ value of heterogeneity (between designs) & $/$ \\
Docetaxel & 1.00 \\
OR & 26.5 \\
P-score (\%) & \\
Anti-PD-1 & $0.36(0.25-0.52)$ \\
OR (95\% CI) & 99.9 \\
P-score (\%) & \\
Anti-PD-L1 & $1.02(0.62-1.66)$ \\
OR (95\% CI) & 23.5 \\
P-score (\%) & \\
\hline Abbreviations: PD-1 = programmed death 1; PD-L1 = programmed death ligand 1; \\
OR = odds ratio; CI = confidence interval. \\
a The comparison for this endpoint was responders vs. non-responders.
\end{tabular}

Table 4. Number of patients experiencing overall grade 3-5 toxicities in each study.

\begin{tabular}{lllll}
\hline Study & \multicolumn{2}{l}{ Experimental group } & \multicolumn{2}{l}{ Control group } \\
\hline & No. of grade & Total & No. of grade & Total \\
& $3-4$ & patients & $3-4$ & patients \\
Brahmer 2015 & $9(6.8 \%)$ & 131 & $71(55.0 \%)$ & 129 \\
Borghaei 2015 & $30(10.5 \%)$ & 287 & $144(53.7 \%)$ & 268 \\
Herbst 2015 (arm 1) & $43(12.7 \%)$ & 339 & $109(35.3 \%)$ & 309 \\
Herbst 2015 (arm 2) & $55(16.0 \%)$ & 343 & $109(35.3 \%)$ & 309 \\
Fehrenbacher 2016 & $16(11.3 \%)$ & 142 & $52(38.5 \%)$ & 135 \\
Rittmeyer 2017 & $90(14.8 \%)$ & 609 & $248(42.9 \%)$ & 578 \\
\hline a Arm 1: pembrolizumab 2 $\mathrm{mg} / \mathrm{kg}$; Arm 2: pembrolizumab 10mg $/ \mathrm{kg}$.
\end{tabular}

With regard to tumor response, anti-PD-1 treatment achieved significantly higher response rate than that of anti-PD-L1 and docetaxel groups. Notably, the immune-modified RECIST criteria, a little different from the RECIST criteria, was adopted for evaluating tumor response in anti-PD-L1 group. Therefore, it's of great importance to employ the uniform evaluation criteria in future trials. Since the detailed data on toxicity prolife were not available in the anti-PD-L1 group, overall grade 3-5 toxicities were compared. Consistent with the results of a system review [39], our findings also showed that anti-PD-a and anti-PD-L1 immunotherapy produced significantly less grade 3-5 toxicities. Moreover, the toxicities profile did not differ between anti-PD-1 and anti-PD-L1 treatments, indicating that blocking the PD-L1-B7-1 pathway did not bring additional toxicities.

The limitations of this study should be addressed: HRs and corresponding 95\% CIs were mainly extracted from the original studies without access to individualized data, which may produce reporting bias. Moreover, the criteria for evaluating tumor response varied between studies. To minimize these limitations, we set strict inclusion criteria and three investigators independently reviewed and extracted data.

\section{Conclusions}

In summary, our network meta-analysis firstly compares anti-PD-1 with anti-PD-L1 immunotherapy in patients with locoregionally advanced or metastatic, previously treated NSCLC who failed first-line treatment, and revealed anti-PD-1 treatment was superior to anti-PD-L1 and docetaxel in terms of PFS and tumor response. Moreover, anti-PD-1 could achieved the highest effect on OS, PFS and tumor response. Based on these evidence, it may be more appropriate to choose anti-PD-1 immunotherapy with regard to treatment ranking. However, future head-to-head clinical trials are warranted to confirm the results of current study.

\section{Abbreviations}

NSCLC: non-small cell lung cancer; PD-1: programmed death 1; PD-L1: programmed deathligand 1; OS: overall survival; PFS: progression-free survival; HR: hazard ratio; OR: odds ratio; $\mathrm{CI}$ : confidence interval.

\section{Supplementary Material}

Supplementary methods, figures and tables. http://www.jcancer.org/v09p1200s1.pdf

\section{Acknowledgement}

We sincerely thanked all the reviewers for their kind and helpful comments on this paper.

\section{Competing Interests}

The authors declare no competing financial interests.

\section{References}

1. Travis WD. Pathology of lung cancer. Clin Chest Med. 2011; 32:669-92.

2. Janne PA, Yang JC, Kim DW, et al. AZD9291 in EGFR inhibitor-resistant non-small-cell lung cancer. N Engl J Med. 2015; 372:1689-99.

3. Mok TS, Wu YL, Papadimitrakopoulou VA. Osimertinib in EGFR T790M-Positive Lung Cancer. N Engl J Med. 2017; 376:1993-4.

4. Mok TS, Wu YL, Thongprasert S, et al. Gefitinib or carboplatin-paclitaxel in pulmonary adenocarcinoma. N Engl J Med. 2009; 361:947-57.

5. Sequist LV, Soria JC, Goldman JW, et al. Rociletinib in EGFR-mutated non-small-cell lung cancer. N Engl J Med. 2015; 372:1700-9.

6. Shaw AT, Kim DW, Mehra R, et al. Ceritinib in ALK-rearranged non-small-cell lung cancer. N Engl J Med. 2014; 370:1189-97.

7. Shaw AT, Kim DW, Nakagawa K, et al. Crizotinib versus chemotherapy in advanced ALK-positive lung cancer. N Engl J Med. 2013; 368:2385-94.

8. Shaw AT, Ou SH, Bang YJ, et al. Crizotinib in ROS1-rearranged non-small-cell lung cancer. N Engl J Med. 2014; 371:1963-71.

9. Fossella FV, DeVore R, Kerr RN, et al. Randomized phase III trial of docetaxel versus vinorelbine or ifosfamide in patients with advanced non-small-cell lung cancer previously treated with platinum-containing chemotherapy regimens. The TAX 320 Non-Small Cell Lung Cancer Study Group. J Clin Oncol. 2000; 18:2354-62.

10. Shepherd FA, Dancey J, Ramlau R, et al. Prospective randomized trial of docetaxel versus best supportive care in patients with non-small-cell lung cancer previously treated with platinum-based chemotherapy. J Clin Oncol. 2000; 18:2095-103.

11. Garassino MC, Martelli O, Broggini M, et al. Erlotinib versus docetaxel as second-line treatment of patients with advanced non-small-cell lung cancer and wild-type EGFR tumours (TAILOR): a randomised controlled trial. Lancet Oncol. 2013; 14:981-8. 
12. Hanna N, Shepherd FA, Fossella FV, et al. Randomized phase III trial of pemetrexed versus docetaxel in patients with non-small-cell lung cancer previously treated with chemotherapy. J Clin Oncol. 2004; 22:1589-97.

13. Borghaei H, Paz-Ares L, Horn L, et al. Nivolumab versus Docetaxel in Advanced Nonsquamous Non-Small-Cell Lung Cancer. N Engl J Med. 2015; 373:1627-39.

14. Brahmer J, Reckamp KL, Baas P, et al. Nivolumab versus Docetaxel in Advanced Squamous-Cell Non-Small-Cell Lung Cancer. N Engl J Med. 2015; 373:123-35.

15. Fehrenbacher L, Spira A, Ballinger M, et al. Atezolizumab versus docetaxel for patients with previously treated non-small-cell lung cancer (POPLAR): a multicentre, open-label, phase 2 randomised controlled trial. Lancet. 2016; 387:1837-46.

16. Garon EB, Rizvi NA, Hui R, et al. Pembrolizumab for the treatment of non-small-cell lung cancer. N Engl J Med. 2015; 372:2018-28.

17. Herbst RS, Baas P, Kim DW, et al. Pembrolizumab versus docetaxel for previously treated, PD-L1-positive, advanced non-small-cell lung cancer (KEYNOTE-010): a randomised controlled trial. Lancet. 2016; 387:1540-50.

18. Herbst RS, Soria JC, Kowanetz M, et al. Predictive correlates of response to the anti-PD-L1 antibody MPDL3280A in cancer patients. Nature. 2014; 515:563-7.

19. Rittmeyer A, Barlesi F, Waterkamp D, et al. Atezolizumab versus docetaxel in patients with previously treated non-small-cell lung cancer (OAK): a phase 3, open-label, multicentre randomised controlled trial. Lancet. 2017; 389:255-65.

20. Reck M, Rodriguez-Abreu D, Robinson AG, et al. Pembrolizumab versus Chemotherapy for PD-L1-Positive Non-Small-Cell Lung Cancer. N Engl J Med. 2016; 375:1823-33.

21. Chen DS, Irving BA, Hodi FS. Molecular pathways: next-generation immunotherapy--inhibiting programmed death-ligand 1 and programmed death-1. Clin Cancer Res. 2012; 18:6580-7.

22. Powles T, Eder JP, Fine GD, et al. MPDL3280A (anti-PD-L1) treatment leads to clinical activity in metastatic bladder cancer. Nature. 2014; 515:558-62.

23. Butte MJ, Keir ME, Phamduy TB, et al. Programmed death- 1 ligand 1 interacts specifically with the B7-1 costimulatory molecule to inhibit $\mathrm{T}$ cell responses. Immunity. 2007; 27:111-22.

24. Park JJ, Omiya R, Matsumura Y, et al. B7-H1/CD80 interaction is required for the induction and maintenance of peripheral T-cell tolerance. Blood. 2010; 116:1291-8.

25. Paterson AM, Brown KE, Keir ME, et al. The programmed death-1 ligand 1:B7-1 pathway restrains diabetogenic effector T cells in vivo. J Immunol. 2011; 187:1097-105.

26. Yang J, Riella LV, Chock S, et al. The novel costimulatory programmed death ligand 1/B7.1 pathway is functional in inhibiting alloimmune responses in vivo. J Immunol. 2011; 187:1113-9.

27. Jadad AR, Moore RA, Carroll D, et al. Assessing the quality of reports of randomized clinical trials: is blinding necessary? Control Clin Trials. 1996; 17:1-12.

28. Rucker G. Network meta-analysis, electrical networks and graph theory. Res Synth Methods. 2012; 3:312-24.

29. [Internet] Rucker G, Schwarzer G, Krahn U, et al. Netmeta: Network meta-analysis using frequentist methods. http://cran.r-project.org/web/ packages/netmeta/index.html.

30. Rucker G, Schwarzer G. Ranking treatments in frequentist network meta-analysis works without resampling methods. BMC Med Res Methodol. 2015; 15:58.

31. Chaimani A, Higgins JP, Mavridis D, et al. Graphical tools for network meta-analysis in STATA. PLoS One. 2013; 8:e76654.

32. Salanti G, Ades AE, Ioannidis JP. Graphical methods and numerical summaries for presenting results from multiple-treatment meta-analysis: an overview and tutorial. J Clin Epidemiol. 2011; 64:163-71.

33. Blanchard P, Hill C, Guihenneuc-Jouyaux C, et al. Mixed treatment comparison meta-analysis of altered fractionated radiotherapy and chemotherapy in head and neck cancer. J Clin Epidemiol. 2011; 64:985-92.

34. Chen YP, Wang ZX, Chen L, et al. A Bayesian network meta-analysis comparing concurrent chemoradiotherapy followed by adjuvant chemotherapy, concurrent chemoradiotherapy alone and radiotherapy alone in patients with locoregionally advanced nasopharyngeal carcinoma. Ann Oncol. 2015; 26:205-11.

35. Ribassin-Majed L, Marguet S, Lee AWM, et al. What Is the Best Treatment of Locally Advanced Nasopharyngeal Carcinoma? An Individual Patient Data Network Meta-Analysis. J Clin Oncol. 2017; 35:498-505.

36. Chen DS, Mellman I. Oncology meets immunology: the cancer-immunity cycle. Immunity. 2013; 39:1-10.

37. Kim JM, Chen DS. Immune escape to PD-L1/PD-1 blockade: seven steps to success (or failure). Ann Oncol. 2016; 27:1492-504.

38. Zou W, Chen L. Inhibitory B7-family molecules in the tumour microenvironment. Nat Rev Immunol. 2008; 8:467-77.

39. Nishijima TF, Shachar SS, Nyrop KA, et al. Safety and Tolerability of PD-1/PD-L1 Inhibitors Compared with Chemotherapy in Patients with Advanced Cancer: A Meta-Analysis. Oncologist. 2017; 22:470-9. 